Service social

\title{
Qui est-ille ? Le respect langagier des élèves non-binaires, aux limites du droit
}

\section{Florence Ashley}

Volume 63, numéro 2, 2017

Genres et sexualités chez les jeunes

URI : https://id.erudit.org/iderudit/1046498ar

DOI : https://doi.org/10.7202/1046498ar

Aller au sommaire du numéro

\section{Éditeur(s)}

École de travail social et de criminologie de l’Université Laval

ISSN

1708-1734 (numérique)

Découvrir la revue

Citer cet article

Ashley, F. (2017). Qui est-ille ? Le respect langagier des élèves non-binaires, aux limites du droit. Service social, 63(2), 35-50. https://doi.org/10.7202/1046498ar
Résumé de l'article

Dans cet article, l'autrice explore la question de savoir si une obligation légale de respecter les pronoms et accords neutres existe en contexte scolaire francophone au Québec. Ille débute par un survol du contexte vécu par les personnes non-binaires francophones ainsi que de leurs besoins par rapport au respect des pronoms et accords neutres. Ensuite, ille analyse l'état actuel du droit relativement au harcèlement et à la discrimination envers les personnes trans en contexte scolaire et évalue la plausibilité qu'une obligation de respecter les pronoms et accords neutres soit retenue par les tribunaux québécois. Enfin, à la lumière des limites du droit, ille propose une approche stratégique concernant le respect des pronoms et accords neutres qui met l'accent sur le développement de politiques institutionnelles s'appuyant sur le droit. 


\title{
Qui est-ille ? Le respect langagier des élèves non-binaires, aux limites du droit
}

\author{
ASHLEY, Florence \\ Candidate au LL.M. à l'Université McGill
}

\section{RÉSUMÉ}

Dans cet article, l'autrice explore la question de savoir si une obligation légale de respecter les pronoms et accords neutres existe en contexte scolaire francophone au Québec. Ille débute par un survol du contexte vécu par les personnes non-binaires francophones ainsi que de leurs besoins par rapport au respect des pronoms et accords neutres. Ensuite, ille analyse l'état actuel du droit relativement au harcèlement et à la discrimination envers les personnes trans en contexte scolaire et évalue la plausibilité qu'une obligation de respecter les pronoms et accords neutres soit retenue par les tribunaux québécois. Enfin, à la lumière des limites du droit, ille propose une approche stratégique concernant le respect des pronoms et accords neutres qui met l'accent sur le développement de politiques institutionnelles s'appuyant sur le droit.

Mots-clés : personnes non-binaires ; transgenre ; droit ; éducation ; langage non genré

\begin{abstract}
In this article, the author explores the question of whether there exists a legal obligation to respect gender neutral pronouns and accordance in schools in Quebec. They begin with an overview of the lived context of non-binary francophone people as well as of their needs with regards respect for gender neutral pronouns and accordance. Then, they analyse the current state of the law on harassment and discrimination against trans people in schools and evaluate the plausibility that an obligation to respect gender neutral pronouns and accordance would be recognised by Quebec courts. Finally, in light of the limits of law, they propose a strategic approach to the respect of gender neutral pronouns and accordance that puts the focus on the developing of institutional policies informed by the law.
\end{abstract}

Keywords: non-binary people; transgender; law; education; gender neutral language 


\section{INTRODUCTION}

La reconnaissance du genre est un signe de respect, en sociétés euro-américaines. Pensons à la série télévisée états-unienne Scrubs. Dans cette série, le Dr Perry Cox se plaît à mégenrer ${ }^{1}$ le $\mathrm{Dr}$ John Dorian - un homme cisgenre ${ }^{2}$. Perry Cox démontre ainsi son dédain pour John Dorian à coups de pronoms traditionnellement féminins et de prénoms tout aussi habituellement féminins. Loin d'être banale, cette pratique montre le Dr Cox comme une personne cruelle qui harcèle ses employés et crée un environnement de travail toxique pour l'amusement du public. Heureusement, ce n'est qu'un personnage fictif.

Pour les personnes non-binaires ${ }^{3}$, le mégenrage est souvent la norme. Malgré le respect érigé en principe dans les milieux scolaires et malgré les avancées sociales sur le plan des droits trans, le respect des pronoms et accords neutres demeure un obstacle de taille pour les étudiant.e.s nonbinaires. La méconnaissance de la grammaire non-genrée et des formes épicènes (Veale et al., 2015, p. 70 ; Unique en son genre, 2018, p. 4) ainsi que le manque de consensus sur le sujet dans les communautés trans québécoises ne font qu'aggraver le problème (Unique en son genre, 2018).

Même si l'écriture non-genrée voit une recrudescence d'intérêt ces dernières années (Lacroux, 2017 ; Scali, 2016 ; Greco et Kunert, 2013 ; Académie française, 2017 ; Lessard et Zaccour, 2017 ; Unique en son genre, 2018 ; Alpheratz, 2017a ; Baril, 2017b ; Greco, 2013, p. 5), le français neutre est encore embryonnaire et peu d'intérêt universitaire lui a été réservé. L'anglais, au contraire, peut facilement être utilisé de façon neutre en empruntant le pronom «they ». Peu de travaux académiques s'appliquent à la situation franco-québécoise, les travaux anglophones oubliant leur spécificité linguistique, alors que les travaux francophones s'intéressent peu aux réalités trans (Baril, 2017a, p. 126-127).

Peut-on parler d'une obligation de respecter les pronoms et accords neutres dans un contexte où la majorité des mots sont genrés et où aucune piste de solution ne fait encore l'objet d'un consensus communautaire? Est-ce que les droits des personnes trans sont circonscrits par l'aisance linguistique? Quel rôle ont et peuvent prendre les politiques institutionnelles par rapport au respect des personnes non-binaires ? Dans le présent article, je tenterai de répondre à ces questions.

1 On mégenre une personne si on utilise des termes genrés qui ne sont pas appropriés au genre de la personne. L'usage de mauvais pronoms («il » pour une personne utilisant «ille»), de mauvais accords (« étudiante » pour une personne utilisant les accords masculins) ou de mauvais termes genrés («princesse » pour un homme) peuvent tous constituer une forme de mégenrage (Unique en son genre, 2016).

2 Une personne est cisgenre ou cis si son identité de genre correspond au genre qui lui fut assigné à la naissance. Une personne sera, au contraire, transgenre ou trans si son identité de genre ne correspond pas au genre qui lui fut assigné à la naissance. Certaines personnes non-binaires ne s'identifient pas comme trans. Toutefois, il est généralement admis que la notion de «trans » inclut les personnes non-binaires.

3 Une personne est non-binaire si elle ne s'identifie pas exclusivement comme homme ou comme femme (ICI Radio Canada, 2015 ; Scali, 2016 ; Information Transgenre, 2013). Les identités non-binaires sont variées, incluant notamment l'identité agenre, genderqueer, fluide dans le genre (genderfluid), demi-genre, etc. Le terme « troisième genre » est à éviter puisque les différentes identités non-binaires sont des genres distincts. Pourquoi troisième et non pas soixante-neuvième ? Ça sonne bien plus agréable... 
J'approche le sujet en tant que personne transféminine francophone qui utilise à la fois « elle » et «ille », mais seulement les accords féminins. L'enjeu du respect des pronoms et accords neutres m'est limitrophe, mais ne me touche pas dans toute sa complexité. En ramenant au premier plan le rapport ambigu que j'entretiens avec mon propre genre, je souhaite souligner l'impact du vécu sur l'analyse en droits de la personne qui, nécessairement, fait appel à des connaissances expérientielles lorsque vient le temps d'évaluer la gravité et proportionnalité des actes discriminatoires.

Dans la première section de l'article, j'exposerai le contexte socio-juridique pertinent pour l'évaluation politique et juridique du respect des pronoms et accords neutres. Dans la deuxième section, je tenterai de déterminer quelles sont, à l'heure actuelle, l'existence et les limites de l'obligation juridique de respecter les pronoms et accords neutres. Dans la dernière section, après avoir conclu en l'incertitude des protections légales, je ferai valoir l'importance des politiques institutionnelles pour combler les lacunes du droit et assurer le respect des personnes non-binaires et suggérerai certains ajouts importants aux politiques récentes pour les personnes non-binaires.

\section{CONTEXTE SOCIO-JURIDIQUE}

Dans cette section, je peins un portrait du contexte socio-juridique pertinent pour l'évaluation juridique de l'obligation au respect des personnes non-binaires et pour l'élaboration de politiques adaptées à leurs réalités. Ce portrait comporte deux parties : les conséquences du mégenrage et l'arrière-plan de vulnérabilité psychosociale chez les personnes non-binaires.

Le non-respect des pronoms et accords est fréquent en contexte scolaire (Chamberland et Puig, 2015 , p. 8). Leur respect est une des revendications principales des communautés trans et nonbinaires (Singh, Meng et Hansen, 2013, p. 215). Comme le dit Alexandre Baril, " [c]'est une marque de respect, c'est une marque de reconnaissance et c'est également très important dans le processus de transition pour s'accepter soi-même et de sentir que donc les gens nous acceptent » (Baril, 2017b). La non-reconnaissance du genre perturbe l'identité sociale et est ressentie comme une injure psychologique (Scali, 2016 ; McLemore, 2015, p. 51, 57 ; McLemore, 2016, p. 2, 7 ; Proulx, 2015). Sa haute fréquence contribue fortement à l'anxiété et à la dépression (McLemore, 2015, p. 52 ; McLemore, 2016, p. 8, 10).

Le sentiment de stigmatisation lié au mégenrage ne semble pas moindre chez les personnes nonbinaires (McLemore, 2015, p. 57), qui représentent environ $36 \%$ des jeunes trans (Veale et al., 2015, p. 14).

D'autres formes de non-respect ou encore de violence sont fréquentes: $56 \%$ des jeunes trans répondant au questionnaire de Galantino et al. (2017, p. 5) avaient, dans les 6 mois précédents, vu leur réputation attaquée, $52 \%$ avaient été victimes d'insultes et de moqueries et $17 \%$ avaient été victimes de violence physique. Dans une autre étude, $87 \%$ des jeunes trans ont rapporté avoir été victimes de harcèlement verbal dans la dernière année (Chamberland, Baril et Duchesne, 2011, p. 4, 12 ; voir aussi Veale et al., 2015, p. 60, 62). Plus de $46 \%$ des jeunes avaient manqué au moins une journée d'école dans le dernier mois à cause notamment du harcèlement et du manque de respect dont illes font l'objet (Chamberland, Baril et Duchesne, 2011, p. 4-5, 20 ; voir aussi Chamberland et Puig, 2015, p. 9).

Certaines études démontrent que l'hostilité et l'insensibilité au genre augmentent les risques de tentatives de suicide et le décrochage scolaire (Goldblum et al., 2012, p. 469, 471 ; Haas, Rodgers et 
Herman, 2014, p.11; Chamberland et Puig, 2015, p. 9). Plus de $19 \%$ des étudiant.e.s trans ontarien.ne-s ont évité l'école par peur d'être harcelée.s ou d'être lu·e.s comme étant trans (Scheim, Bauer et Pyne, 2014, p. 1 ; voir aussi Galantino et al., 2017, p. 14 et Meyer, 2014, p. 72-73).

À mon avis, le respect des pronoms et accords est une des manifestations principales du soutien social dans la vie de tous les jours. II est d'autant plus important de les respecter que les étudiant.e.s trans et non-binaires portent une attention particulière aux signes d'acceptation ou d'intolérance en milieu scolaire (Chamberland et Puig, 2015, p. 10). Le droit moral à l'éducation dépend d'une atmosphère sécuritaire et confortable, ce qui inclut le respect du genre des élèves non-binaires.

Malheureusement, la majorité des institutions scolaires manquent de connaissances au sujet des réalités trans (Chamberland, Baril et Duchesne, 2011, p. 5) et ne sont pas outillées pour faire respecter le genre des jeunes non-binaires. Si Chamberland, Baril et Duchesne ne s'intéressent pas particulièrement aux connaissances des pronoms et accords neutres, la complexité et nouveauté du français neutre laisse à penser que les connaissances sont pires à ce niveau (Unique en son genre, 2018 ; Alpheratz, 2017b ; Alpheratz 2018 ; Lessard et Zaccour, 2017).

Le non-respect des personnes non-binaires est monnaie courante autant qu'il est dévastateur. Si le respect des pronoms et accords neutres vient trop peu et trop tard, est-ce que le droit pourrait obliger à leur respect systématique ? C'est cette question vers laquelle je me tourne à présent.

\section{Analyse}

Dans cette section, j'analyserai la portée du droit au respect des pronoms et accords neutres du point de vue du droit à la vie privée, de la prohibition du harcèlement et du droit à l'égalité. Je conclurai que ces protections sont limitées et ont une portée incertaine par rapport aux accords.

\section{Motifs de discrimination}

Les personnes trans sont protégées par la Charte des droits et libertés de la personne, RLRQ c. C-12 ("Charte québécoise "). Avant l'ajout de l'identité et l'expression de genre à la Charte québécoise par le projet de loi 103 (Loi visant à renforcer la lutte contre la transphobie et à améliorer notamment la situation des mineurs transgenres, LQ 2016, c. 19), la protection des personnes non-binaires était incertaine. Si les personnes trans étaient protégées sous le terme "sexe " depuis la décision Commission des droits de la personne et des droits de la jeunesse c. Maison des jeunes A..., [1998] RJQ 2549 ; 33 CHRR 263, Me Jean-Sébastien Sauvé note qu'il n'est pas certain que toutes les personnes trans soient protégées contre la discrimination par cet arrêt, les personnes non-binaires ne rentrant pas aussi facilement dans la terminologie binaire de "sexe " telle qu'utilisée en droit québécois (Sauvé, 2015).

Depuis l'ajout de l'identité et l'expression de genre, l'article 10 de la Charte se lit ainsi : "Toute personne a droit à la reconnaissance et à l'exercice, en pleine égalité, des droits et libertés de la personne, sans distinction, exclusion ou préférence fondée sur [...] l'identité ou l'expression de genre [...]. » Indépendamment de l'intention législative, qui ne semble pas être dirigée vers la protection des personnes non-binaires, ce changement a pour effet d'inclure explicitement celles-ci dans les protections de la Charte québécoise. Les protections de l'État ayant été accordées aux personnes 
non-binaires, leur statut juridique est dorénavant moins précaire, même si les protections formelles ne présagent pas toujours une protection substantive (Ashley, 2018).

\section{Atteintes aux droits de la personne}

L'obligation de respect des pronoms et accords se conçoit sous trois aspects : le droit à la vie privée, la prohibition du harcèlement et le droit à l'égalité.

\section{DROIT À LA VIE PRIVÉE}

Le droit à la vie privée offre une protection contre le dévoilement du genre assigné à la naissance d'une personne non-binaire. Toutefois, cette protection est inefficace lorsque le genre assigné à la naissance est connu des autres ou encore lorsque le mégenrage ne dévoile pas le genre assigné à la naissance. Le droit à la vie privée n'offre donc pas une garantie efficace du respect des pronoms et accords neutres.

«Toute personne a droit au respect de sa vie privée. » «Toute personne est titulaire de droits de la personnalité, tels le droit [...] au respect [...] de sa vie privée. » Voilà les termes employés par l'article 5 de la Charte québécoise et l'article 3 du Code civil du Québec, RLRQ c. CCQ-1991. Le privé nonbinaire se conçoit principalement en termes de confidentialité de l'assignation de genre à la naissance, confidentialité du morinom - nom attribué à la naissance - et confidentialité par rapport à l'anatomie.

La confidentialité, ramenée au droit, se rapporte à ce qu'on appelle le droit à l'anonymat (Glenn, 1979, p. 881). Dans le spectre du droit à la vie privée, l'usage de pronoms et accords qui révéleraient l'assignation de genre à la naissance d'une personne non-binaire serait une atteinte au droit à l'anonymat. La diffusion d'informations relatives à l'état de santé ou à l'anatomie, celles relatives à l'orientation sexuelle ou encore celles relatives à la transitude ${ }^{4}$ sont toutes comprises dans le droit à l'anonymat (Valiquette c. The Gazette, [1997] R.J.Q. 30 (C.A.), para. 28 [« Valiquette »]). Toutefois, les pronoms et accords neutres dévoilent la transitude dans la mesure où une personne non-binaire n'est pas cisgenre. De façon générale, le droit à l'anonymat ne protégera donc pas une personne nonbinaire contre le dévoilement de sa transitude, même lorsque l'attention est portée sur celle-ci à cause d'une situation de mégenrage. Le mégenrage peut, toutefois, dévoiler l'assignation faite à la naissance et la forme des caractéristiques sexuelles primaires et secondaires - ouvrant la porte au harcèlement de la part des autres élèves.

Le droit à la vie privée demeure néanmoins très limité. II est difficile d'avoir une attente légitime par rapport au caractère confidentiel de l'assignation de genre à la naissance si celle-ci est visible pour le grand public. Le droit à la vie privée est aussi privé de son mordant dans le cas des personnes nonbinaires qui, contrairement aux personnes trans binaires, publicisent leur transitude en communiquant leur genre non-binaire.

Les maux causés par le non-respect des pronoms et accords proviennent plutôt de l'invalidation du genre. Le droit à la vie privée est donc peu adapté à la condamnation de ce non-respect. On devine

\footnotetext{
${ }^{4}$ Le fait d'être trans : Office québécois de la langue française, 2017.
} 
ainsi que les droits à la protection contre le harcèlement et à l'égalité seront plus aptes à cerner et punir le transantagonisme ${ }^{5}$ inhérent au non-respect des pronoms et accords neutres.

\section{PROHIBITION DU HARCÈLEMENT}

La prohibition du harcèlement est large et interdit vraisemblablement le non-respect intentionnel et ou répétitif des pronoms et accords neutres. Comme nous le verrons dans la section suivante, le fait que le mégenrage peut constituer une forme de harcèlement fait intervenir la protection du droit à l'égalité.

Le harcèlement d'une personne en raison de son identité ou expression de genre est interdit par la Charte québécoise. L'article 10.1 nous dit, en effet, que « [n]ul ne doit harceler une personne en raison de l'un des motifs visés dans l'article 10 », motifs qui incluent l'identité et l'expression de genre. L'article 10.1 complémente le droit à l'égalité édicté par l'article 10.

Loi ontarienne, le Code des droits de la personne (L.R.O. 1990, chap. H. 19, art. 101(1)) définit le harcèlement comme le «[f]ait pour une personne de faire des remarques ou des gestes vexatoires lorsqu'elle sait ou devrait raisonnablement savoir que ces remarques ou ces gestes sont importuns ». Cette définition illustre bien la notion de harcèlement en droit québécois, la Charte québécoise ne définissant pas le harcèlement. II n'est pas nécessaire que l'intention de harceler existe pour qu'il y ait harcèlement (Vanderputten v. Seydaco Packaging Corp., 2012 HRTO 1977 [《Vanderputten »], para. 77 ; Charte québécoise, art. 49). II suffira de démontrer une conduite vexatoire ou non désirée dont la fréquence et gravité entraînent un effet durable (De Gaston c. Wojcik, 2012 QCTDP 20, JE 2012-548 [«Gaston »], para. 70).

Comme le soulignent aussi bien le tribunal dans Gaston que la Commission ontarienne des droits de la personne (CODP), c'est le point de vue de la victime non-binaire raisonnable qui sera pertinent pour juger si une conduite constitue du harcèlement (Gaston, para. 71 ; CODP, 2014, p. 19-20). À la lumière des normes sociales et linguistiques dominantes, il sera difficile de prouver le harcèlement si la personne accusée ne s'est pas informée et n'a pas été informée de l'identité de genre ou des pronoms et accords utilisés par la personne non-binaire. Le mégenrage occasionnel sera vraisemblablement excusé par la loi compte tenu du critère de fréquence intrinsèque à la définition de harcèlement.

Compte tenu de la conception qu'ont les tribunaux du mégenrage, il est fort probable que celui-ci puisse constituer du harcèlement. Par exemple, décrire une personne sur la base de son genre assigné à la naissance est considéré être une violation du droit à la dignité (Vanderputten, para. 66, 71 ) et dans $X Y$ v. Ontario (Government and Consumer Services) (2012 HRTO 726 [« XY»], para. 171-172), le tribunal accorde un poids considérable au fait qu'un document d'identité non concordant invalide l'identité de la personne trans et suggère que cette identité n'est pas suffisamment légitime. Les mêmes effets sont présents dans le non-respect des pronoms et accords neutres.

\footnotetext{
5 Le terme «transantagonisme » est proposé à la place du terme plus commun «transphobie » dans la mesure où ce dernier place l'accent étymologique sur le rapport émotif (" phobie ») aux personnes trans, passant sous silence l'intégration de ces attitudes et comportements dans une structure sociale hostile aux personnes trans. Pour une discussion du terme «transantagonisme » en contexte anglophone, voir Ashley, 2018, p. 3.
} 
Compte tenu de la désobligeance, voire du caractère injurieux du mégenrage ainsi que de l'atmosphère d'hostilité provoqué par celui-ci, le non-respect des pronoms et accords neutres est une forme de harcèlement tel que défini en droit québécois (voir aussi voir aussi CODP, 2014, p. 20).

\section{DROIT À L'ÉGALITÉ}

Le droit à l'égalité fait office de pont entre la prohibition individuelle du harcèlement et la responsabilité institutionnelle par rapport au non-respect de l'identité de genre non-binaire. L'établissement scolaire pourra être tenu légalement responsable du mégenrage de son personnel scolaire ainsi que des autres élèves, en l'absence d'efforts suffisants pour éradiquer le harcèlement.

La responsabilité de l'établissement peut être engagée par le refus des éducateurices et autre personnel en position d'autorité d'intervenir lorsqu'une personne est mégenrée, que ce soit par un·e adulte ou par un.e élève. Cet aspect de la responsabilité est crucial en milieu éducatif à cause de l'étendue des interactions inter-élèves, et s'articule à travers la notion juridique de milieu empoisonné. La discrimination inhérente au milieu empoisonné relève de l'article 10 de la Charte québécoise, soit le droit à l'égalité. La preuve du milieu empoisonné peut être faite notamment lorsqu'une institution manque à son obligation de fournir un environnement scolaire libre de harcèlement (CODP, 2014, p. 24 ; Ross c. Conseil scolaire du district no 15 du Nouveau-Brunswick, [1996] 1 RCS 825).

La présence d'un milieu empoisonné donne naissance à une obligation d'intervention de la part des personnes responsables (CODP, 2014, p. 25). Sauf justification, la responsabilité de l'établissement scolaire sera engagée par un manquement à cette obligation, les éducateurices et membres du personnel scolaire se devant d'intervenir. Le rôle des enseignant.e.s est essentiel compte tenu de l'obligation de fournir un milieu d'éducation libre de harcèlement, ceux-ci ayant l'accès le plus direct aux étudiant·e.s, ainsi qu'une autorité effective sur celleux-ci.

\section{Justification de l'atteinte}

Les droits de la personne ne sont pas absolus. Une violation de la prohibition de harcèlement ou du droit à l'égalité pourra être justifiée par l'établissement si aucun accommodement raisonnable n'est possible.

L'obligation d'accommodements raisonnables est définie comme « [l]'obligation de prendre des mesures d'accommodement raisonnables en faveur des personnes subissant les effets préjudiciables d'une politique ou d'une règle apparemment neutre » et "cesse lorsqu'elle va entraîner des "contraintes excessives" » (Eldridge c. Colombie-Britannique (Procureur général), [1997] 3 RCS 624, para. 79). La raison d'être de cette obligation est l'existence d'un "ensemble complexe d'obstacles systémiques et apparemment neutres " (Colombie-Britannique (Public Service Employee Relations Commission) c. BCGSEU, [1999] 3 RCS 3 («Meiorin »), para. 42).

La justification d'un obstacle à l'égalité se fait en trois étapes (Conseil des Canadiens avec déficiences c. VIA Rail Canada Inc., [2007] 1 RCS 650 [«VIA Rail »]). Premièrement, l'obstacle doit être lié à un objectif légitime. Deuxièmement, l'obstacle doit être relié à une croyance honnête et sincère que celui-ci est nécessaire à la satisfaction de l'objectif. Troisièmement, l'obstacle doit être raisonnablement nécessaire à la satisfaction de cet objectif. C'est l'analyse de la troisième étape qui contiendra la nécessité d'accommoder jusqu'à la contrainte excessive. Ce sera à l'établissement 
scolaire ou à la commission scolaire de justifier l'obstacle et donc l'absence d'accommodement (CODP, 2014, p. 32 ; $X Y$, para. 232).

La facilitation des rapports sociaux que comporte le respect des normes sociolinguistiques prédominantes et du genre des élèves est vraisemblablement l'objectif justifiant la norme selon laquelle lesélèves et enseignant·e.s ont pour fonction de deviner le genre de la personne. Cet objectif semble légitime. On peut aussi penser que cette norme, laissant aux enseignant.e.s le rôle de " deviner » le genre de l'élève, relève d'une croyance sincère et de bonne foi en sa nécessité. Le caractère erroné de la croyance importe peu légalement puisque le droit se satisfait de toute croyance sincère et de bonne foi. Ce sera plutôt le troisième pan de l'analyse qui formera le noyau contentieux.

Est-ce que l'obstacle est raisonnablement nécessaire à la satisfaction de l'objectif ? Si la réponse est négative, l'obstacle est injustifié. Si la réponse est positive, nous devrons nous demander si un accommodement raisonnable est possible.

Le langage neutre n'est pas bien connu, est difficile à utiliser en permanence, et plusieurs se sentiraient insulté.e.s de se faire genrer au neutre. Plusieurs personnes trans binaires, même, se sentiraient potentiellement invalidé.e.s par cette nouvelle norme. Que nous partagions ou non ces sentiments, leur présence aura un impact important sur l'évaluation qui sera faite de la nécessité de l'obstacle à cette étape de l'analyse. II semble donc que l'objectif soit servi par la norme de laisser les gens choisir leur langage sur la base des apparences.

La question se corse au niveau des accommodements. En contexte anglophone, il est difficile d'arguer que l'accommodement individuel comporterait une contrainte excessive : le respect des personnes non-binaires ne revient qu'à changer les pronoms « he » ou «she » par «they », etc. Si l'habitude de l'usage peut prendre un certain temps à se développer, mes expériences démontrent que le changement demeure très peu exigeant dans l'ensemble. La campagne « No Big Deal », créée par lea professeur.e non-binaire ontarien-ne Lee Airton, est fondée sur l'idée que le respect des pronoms neutres en anglais est facile (Airton, 2016).

Cependant, le français n'est pas l'anglais. Le pronom singulier «they » existe depuis plusieurs centaines d'années, et l'accommodement revient à redéployer l'usage d'un pronom connu. Aucun consensus similaire n'existe en français. Si un consensus semble imminent autour du pronom «iel » (Unique en son genre, 2018), ceux-ci sont des néologismes et, de plus, ne couvrent pas la problématique des accords : c'est la grammaire entière qui doit être révisée ! Ayant moi-même assisté à des ateliers sur le français neutre que j'utilise assez régulièrement en référence à des personnes proches de moi, je remarque malgré tout une certaine lenteur lorsque je l'utilise à l'oral. II ne serait pas très surprenant, alors, que certaines personnes tentent de justifier leur refus d'accommoder par l'alourdissement grammatical engendré par celui-ci.

II semble que l'obligation de respecter les pronoms et accords neutres serait une contrainte pertinente sur le plan légal puisque la facilitation des interactions dans le milieu scolaire est un facteur légitime (Meiorin, para. 63 ; Central Alberta Dairy Pool v. Alberta (Human Rights Commission), [1990] 2 SCR 489 ; Colombie-Britannique (Superintendent of Motor Vehicles) c. Colombie-Britannique (Council of Human Rights), [1999] 3 RCS 868, para. 41 ; VIA Rail, para. 123). Est-elle excessive ? Pour en juger, nous devons prendre en compte la nature, la légitimité et la force des intérêts en question (VIA Rail, para. 127). 
Si je crois fortement que cette contrainte n'est pas excessive, ma perspective en tant que personne trans n'est partagée, à ma connaissance, par aucun·e de nos juges. L'existence d'une obligation de respecter les pronoms et accords neutres demeure ambiguë, se heurtant à l'incertitude du droit. Si le respect des pronoms peut difficilement être jugé excessif, le respect des accords pourrait l'être. En l'absence d'un jugement à portée juridique claire, la portée du droit au respect des pronoms et accords est vouée à l'incertitude.

Le détail de l'accommodement demandé est important : certaines personnes, par exemple, utilisent les pronoms «iel », «ille » ou «al », mais acceptent que les accords soient genrés. D'autres acceptent une hiérarchie flexible d'accords et pronoms, laissant aux personnes éprouvant de bonne foi une difficulté avec le français neutre le choix de genrer leurs accords autrement tout en respectant l'élève non-binaire. Cet accommodement risquera moins d'être jugé comme représentant une contrainte excessive qu'un accommodement demandant le respect des accords non genrés.

L'obligation d'accommodement n'est pas seulement substantive, mais aussi procédurale (CODP, 2014, p. 27). En effet, il revient à l'établissement scolaire d'inclure la personne non-binaire dans les discussions sur les accommodements à instaurer. Cette obligation procédurale est essentielle compte tenu de la variété des choix et des identifications langagières des personnes non-binaires. Une personne utilisant des pronoms neutres pourrait très bien désirer, comme moi, être catégorisée avec les femmes et se reconnaître dans des termes genrés comme «princesse ». II est aussi possible d'utiliser un pronom neutre tout en acceptant des accords genrés. Dans un tel cas, l'obligation de respect des pronoms et accords est claire. Au contraire, la loi est incertaine si des accords neutres sont demandés.

\section{Politiques et Éducation}

En l'absence d'un droit clair et certain, les politiques institutionnelles peuvent jouer un rôle important en promouvant le respect du genre des personnes non-binaires. Celles-ci sont moins chères à établir, peuvent être plus claires, spécifiques et flexibles que le droit et peuvent codifier des normes plus exigeantes que le minimum légal.

Le droit étant incertain par rapport au respect des accords neutres, les politiques institutionnelles devraient comporter des recommandations claires et détaillées par rapport à ce sujet : est-ce que les pronoms et accords neutres doivent être respectés ? par qui ? de quelle façon ?

Plusieurs organismes et institutions ont établi ces dernières années des politiques qui incluent le respect des pronoms des personnes trans. Pensons notamment aux lignes directrices du Ministère de l'Éducation et du Développement de la petite enfance de la Nouvelle-Écosse (2014), aux Lignes directrices relatives au soutien des élèves transgenres de la Commission scolaire de Montréal (2016) ainsi qu'aux plus récentes Mesures d'ouverture et de soutien envers les jeunes trans et les jeunes non-binaires de la Table nationale de lutte contre l'homophobie et la transphobie des réseaux de l'éducation (2017). Plusieurs autres documents portent sur les mesures à prendre pour soutenir les jeunes trans tant en contexte francophone qu'anglophone (Chamberland et Puig, 2015 ; Singh, Meng et Hansen, 2013 ; Beemyn, 2003 ; Taylor et al., 2016 ; Gay-Straight Alliance Network et al., 2004 ; Lambda Legal et National Youth Advocacy Coalition, 2008 ; Vallières, 2015 ; Chamberland, Baril et Duchesne, 2011). 
Comme le remarquent Chamberland et Puig, en l'absence de mesures explicites, les élèves « doivent s'en remettre au personnel pour les accommoder dans l'utilisation de leur nouvelle identité, s'exposant là aussi à l'incompréhension et au rejet » (Chamberland et Puig, 2015, p. 11). II est donc louable que les divers documents cités incluent une reconnaissance du devoir de respecter les pronoms des élèves non-binaires, ainsi que du devoir de systématiser le processus de changement de mention de sexe. Ce dernier devoir peut aller jusqu'à celui d'offrir une troisième option de genre ou d'enlever toute mention de genre sur les dossiers de l'élève, une avancée considérable par rapport à la norme actuelle dans les écoles. Un pas de plus serait d'envisager la possibilité d'inclure systématiquement les pronoms et accords désirés des élèves sur les listes de classes.

Malheureusement, les politiques de la Commission scolaire de Montréal et de la Table nationale sont fortement inspirés de politiques tirées de contextes anglophones - dont notamment le guide de la Fédération canadienne des enseignantes et des enseignants (Wells, Roberts et Allan, 2011). Ce fait conspire avec le manque de personnes non-binaires dans les équipes de rédaction des politiques pour produire un manque d'attention relativement à la problématique des accords neutres en français. Cette dynamique - le manque d'attention à l'égard du français combiné à un manque d'attention à l'égard des identités non-binaires - fait écho aux observations d'Alexandre Baril par rapport aux études féministes francophones (Baril, 2017a), à la différence que le binarisme est centré à la place de l'identité cisgenre.

Tandis que la loi se bute aux difficultés du français neutre, nos politiques institutionnelles refusent de s'attaquer à celles-ci. Ce refus perpétue le manque de protections auquel font face les élèves nonbinaires. Comme le disent Chamberland et Puig, "c'est en s'appuyant sur les lignes directrices et les politiques institutionnelles en place, et sachant que la direction les soutient, que le personnel ainsi que les étudiant.e.s pourront développer des actions à plus petite échelle au sein de l'établissement. [...] II est aussi important de s'attarder à ce que les individus ressentent et aux besoins qu'ils expriment " (2015, p. 17). Ces recommandations méritent suite.

Selon une étude récente de Taylor et al., seulement $24 \%$ des commissions scolaires du Québec ont une politique visant le harcèlement des personnes trans (Taylor et al., 2016, p. 26). Et ces politiques comptent probablement peu de suggestions concernant spécifiquement les jeunes non-binaires. Cependant, on peut penser que plusieurs commissions scolaires emboîteront le pas à la Commission scolaire de Montréal dans les prochaines années et adopteront une politique par rapport aux jeunes trans. Nous sommes donc à un moment critique de la propagation de ces politiques, et il est crucial de souligner l'importance de développer nos politiques par rapport au respect des pronoms et accords neutres, et cela de façon détaillée. Une liste des pronoms les plus communs devrait être donnée : iel, yel, ielle, ael, æl, aël, ol, olle, ille, ul, ulle, al, i, im (Unique en son genre, 2018, p. 12). Les stratégies de neutralisation des accords devraient aussi être exposées, dont notamment les graphies tronquées comme "étudiant.e ", les néologismes et nouvelles graphies comme "elleux » pour "elles » et "eux » et la rédaction épicène comme «l'élève » à la place de "l'étudiant » ou "l'étudiante " (Lessard et Zaccour, 2017 ; Alpheratz, 2017a ; Alpheratz, 2017b ; Alpheratz 2018 ; Unique en son genre, 2018, p. 27).

Dans le développement de futures politiques, on devra s'assurer d'engager plus largement des personnes trans et non-binaires au processus de rédaction de ces politiques. II est critiquable que la Table nationale de lutte contre l'homophobie et la transphobie des réseaux de l'éducation ne semble pas avoir inclus de personnes non-binaires, voire de personnes trans, à la rédaction. Le contenu de 
ces politiques devra aussi refléter la diversité des personnes non-binaires ainsi que reconnaître la difficulté du française neutre. II n'existe pas de formule générale pour respecter toutes les personnes non-binaires. Nos politiques devront être suffisamment souples dans leur énoncé pour pouvoir en prendre acte et s'ajuster selon l'étudiant·e individuel·le.

La mise en œuvre du respect des personnes non-binaires doit prendre en compte les perspectives des personnes trans francophones et des personnes ayant des difficultés d'apprentissage. Tant l'anglocentrisme du langage trans que les difficultés d'apprentissage créent un décalage entre les réalités cisgenres anglophones et les réalités trans francophones.

La notion du « temps trans-crip-t » d'Alexandre Baril (2016) nous force à prendre acte du fait que nous devrons investir des ressources concrètes pour enseigner le français neutre à l'ensemble du corps étudiant québécois, et non pas seulement aux étudiant·e-s ayant des facilités d'apprentissage. II n'est pas suffisant de dire qu'il faut respecter les pronoms et accords des personnes trans. Encore faut-il définir et enseigner le français neutre.

\section{Conclusion}

Sous sa forme actuelle, la Charte québécoise promet plusieurs protections aux personnes trans et non-binaires. Certaines de ces protections suggèrent l'existence d'une obligation de respecter les pronoms et accords neutres s'ils sont demandés par la personne. Toutefois, l'ambiguïté de la situation sur le plan juridique ainsi que l'inaccessibilité des tribunaux rend cette obligation difficile à mettre en $œ u v r e$. Les normes genrées singulières du français créent une asymétrie juridique et factuelle entre les contextes francophone et anglophone, même à l'intérieur d'une seule juridiction. II y a un grand besoin de recherches universitaires additionnelles sur la non-binarité et le français neutre.

En l'absence de directives claires de la part des tribunaux, la tendance récente à la création de politiques institutionnelles font apparaître celles-ci comme le recours le plus prometteur pour les étudiant-e.s trans et non-binaires. II est essentiel pour les activistes de souligner l'aspect procédural des obligations des établissements scolaires puisqu'il met en évidence le potentiel transformatif de la consultation. Même si son application demeure chétive, le droit peut servir à informer et à motiver ces politiques.

Compte tenu de l'importance du respect des personnes trans et non-binaires pour leur bien-être personnel et pour leur succès scolaire, les récentes lignes directrices de la Commission scolaire de Montréal marquent, malgré leurs imperfections, un tournant dans l'accès à l'éducation des personnes trans. Espérons que d'autres établissement et commissions scolaires emboîteront le pas.

ASHLEY, Florence

Candidate au LL.M. à l'Université McGill

\section{REMERCIEMENTS}

L'autrice remercie Marie-Philippe Drouin, Jess de Santi et Serena Rivard pour leur aide éditoriale. 


\section{RÉFÉRENCES}

Académie française (2017). "Déclaration de l'Académie française sur l'écriture dite "inclusive" ", 26 octobre 2017. En ligne: <http://www.academie-francaise.fr/actualites/declaration-de-lacademiefrancaise-sur-lecriture-dite-inclusive>

Airton, L. (2016). "What is the NBD Campaign? ", No Big Deal - l'll Use Your Pronoun. site Web : $<$ https://www.nbdcampaign.ca/what/>

Alpheratz (2018). Grammaire du français inclusif, Châteauroux, France, Éditions Vent Solars.

Alpheratz (2017a). "Le genre grammatical neutre en français à la lumière des Problèmes de linguistique générale d'Émile Benveniste ». En ligne : <https://www.academia.edu/33427792/>

Alpheratz (2017b). "Un genre neutre pour la langue française ». En ligne: <https://www.academia.edu/33035651/>

Ashley, F. (2018). " Don't be so hateful: The insufficiency of anti-discrimination and hate crime laws in improving trans well-being ", University of Toronto Law Journal, vol. 68, n 1, p. 1-36.

Baril, A. (2017a). "Intersectionality, lost in translation? (Re)thinking inter-sections between Anglophone and Francophone intersectionality ", Atlantis: Critical Studies in Gender, Culture \& Social Justice, vol. 38, n 1, p. 125-137.

Baril, A. (2017b). «Pour ou contre l'ajout d'un genre neutre à la langue française ? », TFO 24.7, 6 octobre 2017. En ligne : <https://www.youtube.com/watch ?v=2NFWHVBfsY4>

Baril, A. (2016). " "Doctor, am I an Anglophone trapped in a Francophone body?": An intersectional analysis of "trans-crip-t-time" in ableist, cisnormative, and Anglonormative societies ", Journal of Literary \& Cultural Disability Studies, vol. 10, n 2, p. 155-172.

Beemyn, G. (2003). "Serving the needs of transgender college students ", Journal of Gay and Lesbian Issues in Education, vol. 1, n 1, p. 33-50.

Central Alberta Dairy Pool v. Alberta (Human Rights Commission), [1990] 2 SCR 489.

Chamberland, L., A. Baril et N. Duchesne (2011). La transphobie en milieu scolaire au Québec. Rapport de recherche, Université du Québec à Montréal, Montréal.

Chamberland, L., et A. Puig (2015). Guide des pratiques d'ouverture à la diversité sexuelle et de genre en milieu collégial et universitaire, Chaire de recherche sur l'homophobie, Université du Québec à Montréal, Montréal.

Charte des droits et libertés de la personne, RLRQ c. C-12.

Code civil du Québec, RLRQ c. CCQ-1991. 
Code des droits de la personne, L.R.O. 1990, chap. H. 19.

Colombie-Britannique (Public Service Employee Relations Commission) c. BCGSEU, [1999] 3 RCS 3.

Colombie-Britannique (Superintendent of Motor Vehicles) c. Colombie-Britannique (Council of Human Rights), [1999] 3 RCS 868.

Commission des droits de la personne et des droits de la jeunesse c. Maison des jeunes A..., [1998] RJQ 2549 ; 33 CHRR 263.

Commission ontarienne des droits de la personne (2014). Politique sur la prévention de la discrimination fondée sur l'identité sexuelle et l'expression de l'identité sexuelle.

Commission scolaire de Montréal (2016). Lignes directrices relatives au soutien des élèves transgenres de la Commission scolaire de Montréal.

Conseil des Canadiens avec déficiences c. VIA Rail Canada Inc., [2007] 1 RCS 650.

De Gaston c. Wojcik, 2012 QCTDP 20, JE 2012-548.

Eldridge c. Colombie-Britannique (Procureur général), [1997] 3 RCS 624.

Galantino, G., M. Blais, M. Hébert et F. Lavoie (2017). Un portrait de l'environnement social et de l'adaptation psychosociale des jeunes québécois.e.s trans ou en questionnement de leur identité de genre. Rapport de recherche du projet Parcours amoureux des jeunes LGBT au Québec, Université du Québec à Montréal, Montréal.

Gay-Straight Alliance Network, Transgender Law Center et National Center for Lesbian Rights (2004). Beyond the Binary: A Tool Kit for Gender Identity Activism in Schools, Oakland, CA. En ligne : <https://translaw.wpengine.com/wp-content/uploads/2012/07/99640173-Beyond-the-Binary.pdf>

Glenn, H. P. (1979). «Le droit au respect de la vie privée », Revue du Barreau, vol. 39, p. 879.

Goldblum, P., R. J. Testa, S. Pflum, M. L. Hendricks, J. Bradford et B. Bongar (2012). «The Relationship between gender-based victimization and suicide attempts in transgender people ", Professional Psychology: Research and Practice, vol. 43, n 5, p. 468-475.

Greco, L. (2013). “Langage et pratiques "transgenres" », Langues et cité : Bulletin de l'observatoire des pratiques linguistiques, $n^{\circ} 24$, p. 5-6.

Greco, L., et S. Kunert (2013). “Genre, langage et sexualité : Entretien avec Luca Greco », entretien mené par Stéphanie Kunert, Communication et langages, vol. 3, n 177, p. 125-134.

Bauer, G. R., A. I. Scheim, J. Pyne, R. Travers et R. Hammond (2015). «Intervenable factors associated with suicide risk in transgender persons: A respondent driven sampling study in Ontario, Canada », BMC Public Health, vol. 15, n525, p. 1-15. 
Haas, A. P., P. L. Rodgers et J. L. Herman (2014). Suicide Attempts Among Transgender and Gender Non-Conforming Adults, Los Angeles, The Williams Institute.

ICI Radio-Canada (2016). " Petit lexique de l'identité sexuelle », ICI Radio-Canada, 3 décembre 2015. En ligne : <http://ici.radio-canada.ca/nouvelle/752595/lexique-genres-identite-sexuelle>

James, S. E., J. L. Herman, S. Rankin, M. Keisling, L. Mottet et M. Anafi (2016). The Report of the 2015 U.S. Transgender Survey, Washington, National Center for Transgender Equality.

Lacroux, M. (2017). "Prêt.e.s à utiliser l'écriture inclusive ? ", Libération, 27 septembre 2017. En ligne : <http://www.liberation.fr/france/2017/09/27/pretes-a-utiliser-I-ecriture-inclusive_1598867>

Lambda Legal et National Youth Advocacy Coalition (2008). Bending the Mold: An Action Kit for Transgender Youth, New York. En ligne: $<$ https://www.lambdalegal.org/sites/default/files/publications/downloads/btm_bending-themold_0.pdf>

Lessard, M., et S. Zaccour (2017). Grammaire non sexiste de la langue française. Le masculin ne l'emporte plus !, Saint-Joseph-du-Lac, M Éditeur.

Loi visant à renforcer la lutte contre la transphobie et à améliorer notamment la situation des mineurs transgenres, LQ 2016, c. 19.

McLemore, K. A. (2015). " Experiences with misgendering: Identity misclassification of transgender spectrum individuals ", Self and ldentity, vol. 14, $\mathrm{n}^{\circ}$ 1, p. 51-74.

McLemore, K. A. (2016). " A minority stress perspective on transgender individuals' experiences with misgendering ", Stigma and Health. Publication anticipée en ligne : <http://dx.doi.org/10.1037/sah0000070>

Meyer, E. J. (2014). "Supporting gender diversity in schools: Developmental and legal perspectives ", dans A. Pullen-Sansfaçon et E. J. Meyer (dir.), Supporting Gender Creative and Transgender Youths: Family, schools and community in action, New York, Peter Lang.

Ministère de la Sécurité communautaire et des Services correctionnels (2015). Politique ontarienne en matière d'admission, de classification et de placement des détenus et détenues trans, Ontario.

Ministère de l'Éducation et du Développement de la petite enfance (2014). Lignes directrices pour le soutien aux élèves transgenres et non conformistes de genre, Nouvelle-Écosse.

Office québécois de la langue française (2017). "Transidentité", Le grand dictionnaire terminologique. En ligne : <http://www.gdt.oqlf.gouv.qc.ca/ficheOqlf.aspx ?ld_Fiche=8359852>

Proulx, G. (2015). « Un français plus neutre : utopie ? », ICI Radio-Canada Estrie, 3 décembre 2015. En ligne: <http://ici.radio-canada.ca/nouvelle/752820/identites-sexuelles-trans-genres-francaisdifficultes> 
Ross c. Conseil scolaire du district no 15 du Nouveau-Brunswick, [1996] 1 RCS 825.

Sauvé, J.-S. (2015). "L'interdiction de discriminer les personnes trans* dans la Charte des droits et libertés de la personne : pour son amélioration par l'ajout de l'“identité de genre" et de l'“expression de genre" à la liste des motifs de distinction illicites ", Enfances, Familles, Générations, vol. 23, p. 108-126.

Scali, D. (2016). « Ni madame ni monsieur. Des personnes trans revendiquent le droit d'être définies comme "non-binaires dans le genre" », Journal de Montréal, 20 juin 2016. En ligne: <http://www.journaldemontreal.com/2016/06/20/ni-madame-ni-monsieur>

Sébille c. Photo Police, [2007] R.R.A. 320 (C.S.).

Singh, A. A., S. Meng et A. Hansen (2013). " "It's already hard enough being a student”: Developing affirming college environments for trans youth », Journal of LGBT Youth, vol. 10, n 3, p. 208-223.

Table nationale de lutte contre l'homophobie et la transphobie des réseaux de l'éducation (2017). «Mesures d'ouverture et de soutien envers les jeunes trans et les jeunes non-binaires : Guide pour les établissements d'enseignement », Montréal. En ligne : <http://www.colloquehomophobie.org/wpcontent/uploads/2017/11/brochure_jeunes_trans_web-2.pdf>

Taylor, C., T. Peter, T. Edkins, C. Campbell, G. Émond et E. Saewyc (2016). Inventaire national des interventions des commissions scolaires pour soutenir le bien-être des élèves LGBTQ, Stigma and Resilience Among Vulnerable Youth Centre, Université de la Colombie-Britannique, Vancouver.

Unique en son genre (2018). Le langage dans la communauté non-binaire 2017. En ligne: <http://ekladata.com/Gik8DdzqEfaGDTkRfE1qrPz|450/Le-langage-dans-la-communaute-nonbinaire-2017-Unique-en-son-genre.pdf>

Unique en son genre (2016). «Mégenrer c'est violent », blog: <http://uniqueensongenre.eklablog.fr/megenrer-c-est-violent-a119698416>

Valiquette c. The Gazette, [1997] R.J.Q. 30 (C.A.).

Vallières, A. (2015). « Recherche sur les problématiques liées à la diversité sexuelle à l'Université de Montréal », adoptée à la $513^{e}$ séance du Conseil central, Fédération des associations étudiantes du campus de l'Université de Montréal. En ligne : <http://www.faecum.qc.ca/ressources/avis-memoiresrecherches-et-positions-1/recherche-sur-les-problematiques-liees-a-la-diversite-sexuelle-a-luniversite-de-montreal>

Vanderputten v. Seydaco Packaging Corp., 2012 HRTO 1977.

Veale, J., E. Saewyc, H. Frohard-Dourlent, S. Dobson, B. Clark et le Groupe de recherche de l'enquête canadienne sur la santé des jeunes trans (2015). Être en sécurité, être soi-même. Résultats de l'enquête canadienne sur la santé des jeunes trans. Stigma and Resilience Among Vulnerable Youth Centre, Université de la Colombie-Britannique, Vancouver. 
Wells, K., G. Roberts et C. Allan (2011). Soutien aux élèves transgenres et transsexuels dans les écoles de la maternelle à la $12^{e}$ année. Guide à l'intention des éducatrices et éducateurs, Fédération canadienne des enseignantes et des enseignants, Ottawa.

XYv. Ontario (Government and Consumer Services), 2012 HRTO 726. 